\title{
FPGA Implementation of Multiplier-less CDF-5/3 Wavelet Transform for Image Processing using System-Generator
}

\author{
Rashmi $\mathrm{S}^{1}$, Pradeep S V ${ }^{2}$, Dr. Siva Yellampalli ${ }^{3}$ \\ ${ }^{I}$ M. Tech Student, Department of E.C.E., VTU Extension Centre, UTL Technologies Ltd., India \\ ${ }^{2}$ Assistant Professor, Department of E.C.E., VTU Extension Centre, UTL Technologies Ltd., India \\ ${ }^{3}$ Principal, Department of E.C.E., VTU Extension Centre, UTL Technologies Ltd., India
}

\begin{abstract}
Most of the digital image processing application uses various domain transformation technique to convert time domain information to transform domain which will help to simplify the mathematical modeling. Discrete Wavelet Transform is one of the best transformation techniques. The time-frequency resolution makes this transform sensitive to both time and frequency which will give very good compression and decompression. In this paper, we propose FPGA implementation of multiplier-less CDF-5/3 wavelet transform for image processing application using System-Generator tool.To maintain low area and high frequency we use multiplier-less architecture for CDF-5/3 DWT for our implementation. The VHDL code for multiplier-less structure is fed to system generator tool using standard procedure and synthesis the structure to get the area and frequency.
\end{abstract}

Keywords: CDF-5/3, Digital Filters, FPGA Implementation, Image Processing and Wavelet Transform etc.

\section{Introduction}

Wavelets are the mathematical functions used in image processing and DSP applications. It permits both time and frequency analysis. Wavelet principles are similar to frequency analysis. The concept of wavelet is developed in the $19^{\text {th }}$ century. Generally wavelet transforms can be divided into CWT and DWT. CWT operates for entire translation and DWT operates for selected set of transition. Wavelet transform is improved version of Fourier transform, wavelet transform is a better technique for image compression, because Fourier requires all present and future information related to the signal and it can't observe the frequencies varying with time because it is a function independent. Wavelet can be used in many applications such as in signal processing, it improves the weak signal from the noise. In internet communications wavelets can be used to compress the original image to the maximum extent, and it is also used for the decompression algorithms to recover the image without changes or loss to the original image. It can also be used for communication applications.

\section{Literature Surveys}

In this paper[1] wavelet based on lifting scheme is presented. For better image compression CDF $(2,2)$ integer to integer wavelet transform is used. The code is written in HDL and simulated. The implementation is done using both the FPGA and ASIC. Obtained hardware result can be used for the higher image compression ratio.In paper[2] with high pipelined and low memory, 2D lifting architecture is proposed. The proposed novel consists of two new memory units, one row and one column processor units. For $5 / 3$ filter designing only $4 \mathrm{~N}$ temporal memory is needed. At every stage of the cycle two outputs are produced. The code is written in the HDL and simulated. Implementation is done on FPGA board.Number of slices obtained here is 400 and operates at a frequency of 120MHz.High speed, low power DWT based on lifting scheme is presented [3] here. 2D-DWT architecture uses multipliers and adders it uses more power. Proposed novel uses the other algorithm for BZFAD multiplier which consumes the power. This architecture mainly focused on the power reduction in multipliers. The proposed architecture is implemented using ASIC $130 \mathrm{~nm}$ technology. The multiplier is $65 \%$ faster, $35 \%$ power saving and takes $45 \%$ less area compared to the existing multipliers. Frequency of operation is $200 \mathrm{MHz}$. This paper presents lifting scheme architecture for seven filters [4]. It presents both forward and inverse DWT. Architecture consists of two adders, one shifter and one multiplier. Also it contains two memory units, each memory consists of four banks to get high bandwidth. Proposed novel is implemented in VHDL and frequency of operation is increased compared to other architectures.In this paper [5] recursive and dual scan architecture for 1D and 2D wavelet transform is presented. The paper mainly focused on the complexity reduction in wavelet transform. Here both column and row processor operates at a time. Proposed novel represents higher hardware utilization and computation time is less. It minimizes the memory size for storing the results.Paper presents 5/3 lifting based architecture for both 1D-DWT and 2D-DWT [6]. Lifting algorithms is used to design 1D-DWT, by using this algorithms 2D architecture is designed. Implementation is done on the FPGA board. Proposed novel uses less hardware and number of multipliers required here is less.Wavelet 
transform performs the compression of the image to a greater extent. By using $5 / 3$ filter coefficients high speed discrete wavelet transform is designed [7] with less multipliers which in turn reduces the complexity with performance. Implementation is done on the FPGA board.In this paper [8] discrete wavelet transform for higher order image compression is achieved. The novel is simple in designing as well as area efficient. The main aim of this paper is to reduce the memory size of the on chip and complexity of the hardware. Two dimensional wavelet is implemented on the Sparten-3E kit and obtained the high PSNR ratio.In [9] paper for designing wavelet transforms multipliers are replaced by the ROM, the model is called distributive arithmetic architecture, this is much faster then the simple arithmetic architecture. The proposed novel has good throughput hence it is applicable to all image processing applications.In this paper [10] discrete wavelet transform based on lifting scheme is presented. In the proposed novel they have used new dynamic reconfigurable processor which helps in achieving higher compression ratio and high throughput upto $53 \mathrm{fps}$. Architecture is implemented on the FPGA board which gives good results compared to the other FPGA implementations.DWT plays a major rule in the image compression. In this paper[11] new lifting scheme algorithm is used it gives two coefficients. The implemented 1D-DWT equations uses right shift operations and it minimizes the delay and improves the throughput. Implementation is done on vertex-5 FPGA board, power consumption is $1 \mathrm{~W}$ and frequency of operation is $180 \mathrm{MHz}$. Proposed architecture is applicable to all real time image processing applications.In all the existing systems improvement in memory efficiency is done by reducing the on chip memory words, But in this novel memory efficiency improvement is done by reducing the on chip memory word length [12]. The design of both $5 / 3$ and $9 / 7$ DWT is done here and temporal memory reduction is $17.5 \%$.

\section{System-Generator Architecture}

The multiplier-less architecture [13] is coded using VHDL language and the code is called in System-Generator tool using standard procedure [14]. Xilinx ATLYS (xc6slx45-2csg324) board is used for synthesis.

\subsection{Software Implementation}

In software module implementation the blocks are taken from the System-Generator libraries. First the input is taken from the file. Next one is intensity block which is used to convert RGB color image to gray image. Next block is resize, here whatever the size of the gray image the resize converts it into standard size $256 \times 256$. Next process is frame serialization here the image is transposed and two dimensional image is converted into one dimensional image. The values are serially un-buffered. Frame de-serialization performs the reverse operation of frame serialization. Next gateway I/O block is used which interfaces the all System-Generator library blocks. In Xilinx System-Generator block our main project is exists. Next black box is used which is taken from the System-Generator library. With this any required functionality can be activated. Video viewer block is used for displaying input image and the compressed image. Next system generator is taken from the library. Next process is to double click on the system generator which shows the specifications of the FPGA board. Now complete design has to be saved and run. Within few seconds input image and compressed images can be seen.The Figure 1 shows 1D-DWT and Figure 2 shows the whole System-Generator architecture using 1D-DWT.

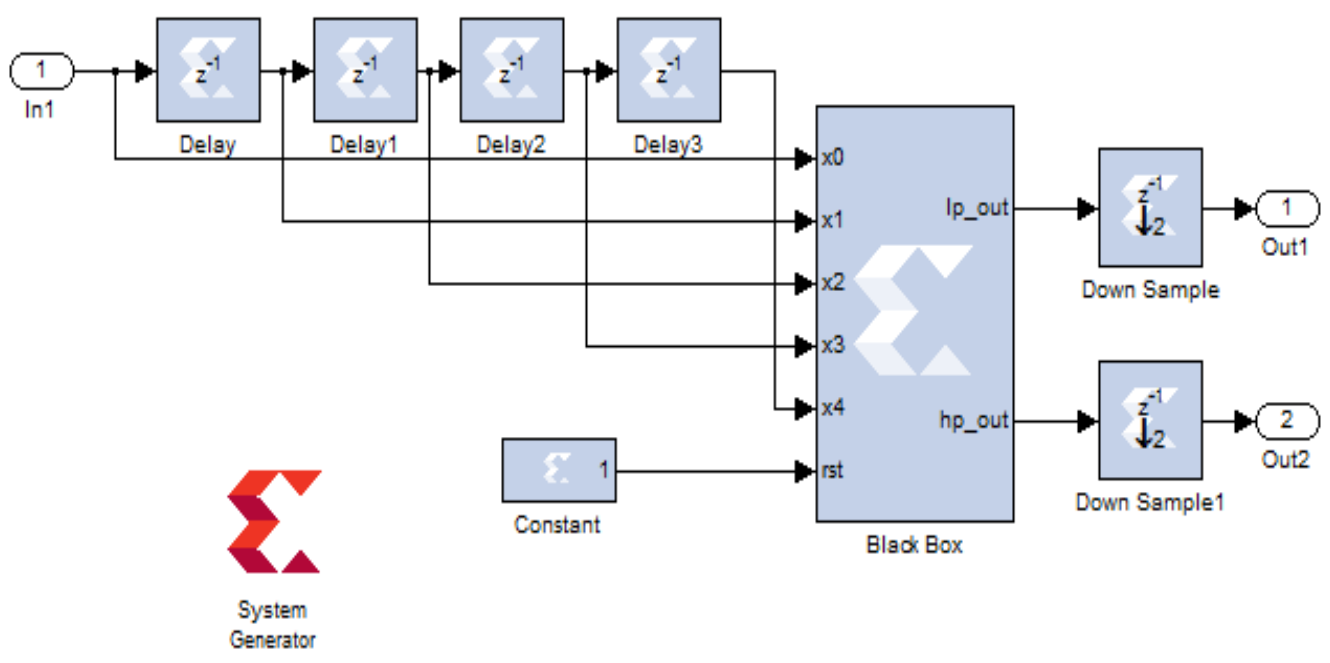

Figure 1: System-Generator Implementation of 1D-DWT 


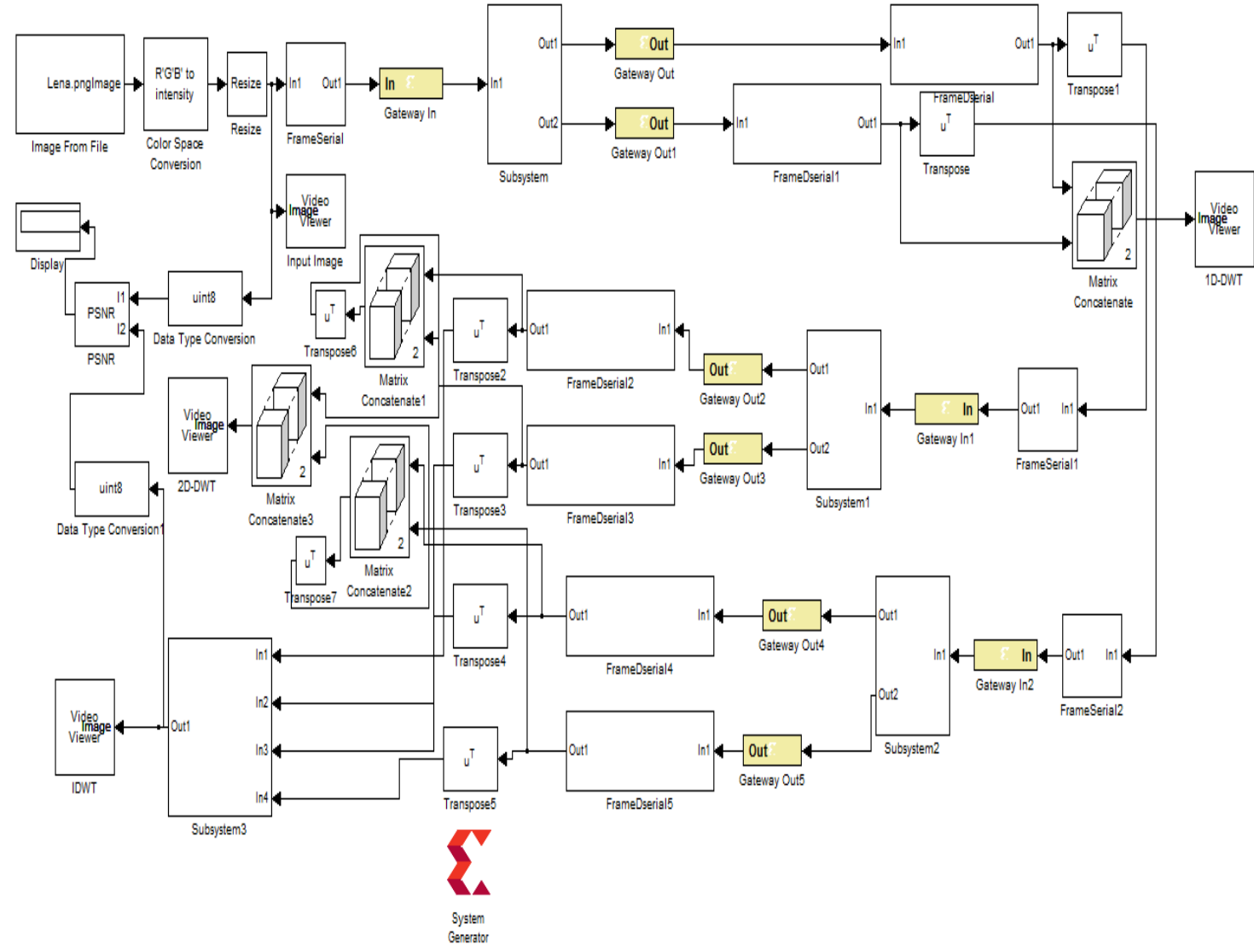

Figure 2: System-Generator Implementation of 2D-DWT

\subsection{Hardware Implementation}

Once the software model results are obtained, next process is to designing the hardware. Here by double clicking on the generate will get the generated hardware design. Next do the connections same like software model. Save the design, make the connections with the FPGA kit. Run the module next output can be seen.

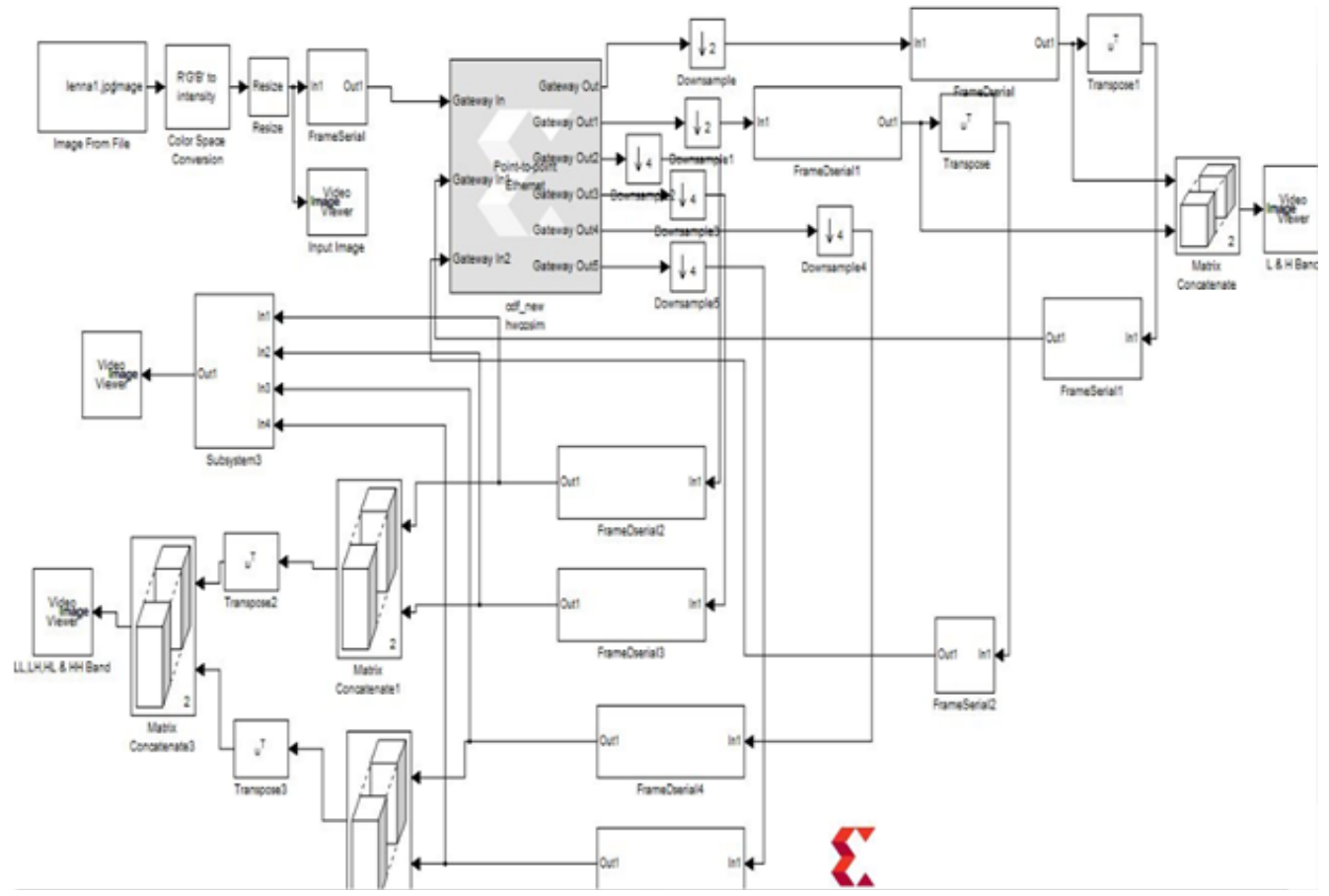

Figure 3: System-Generator Hardware Implementation of 2D-DWT 


\section{Hardware Utilizations}

The hardware utilization of proposed structure is given in Figure.4.

\begin{tabular}{|c|c|c|c|c|}
\hline 2) Compilation status & & & \multicolumn{2}{|c|}{\begin{tabular}{|l|l|l|}
$\square$ & $\square$ & $x$ \\
\end{tabular}} \\
\hline \multicolumn{5}{|l|}{ Compilation finished successfully. } \\
\hline \multicolumn{5}{|l|}{ Slice Logic Utilization: } \\
\hline Number of Slice Registers: & 1,478 out of & 54,576 & 28 & \\
\hline Number used as Flip Flops: & 1,466 & & & \\
\hline Number used as Latches: & 0 & & & \\
\hline Number used as Latch-thrus: & 0 & & & \\
\hline Number used as AND/OR logics: & 12 & & & \\
\hline Number of Slice LUTs: & 1,628 out of & 27,288 & $5 \frac{8}{8}$ & \\
\hline Number used as logic: & 1,579 out of & 27,288 & 5 웅 & \\
\hline Number using 06 output only: & 1,261 & & & \\
\hline Number using 05 output only: & 56 & & & \\
\hline Number using 05 and 06 : & 262 & & & $\square$ \\
\hline Number used as ROM: & 0 & & & \\
\hline Number used as Memory: & 8 out of & 6,408 & 18 & \\
\hline \multirow[t]{2}{*}{ Number used as Dual Port RAM: } & 0 & & & - \\
\hline & OK & Cancel & \multicolumn{2}{|c|}{ Hide Details } \\
\hline
\end{tabular}

Figure 4: Hardware Utilization of 2D-DWT

\section{Image Output}

This section we will give the image output produced by the architecture in Figure 5 where Lena image is considered as input
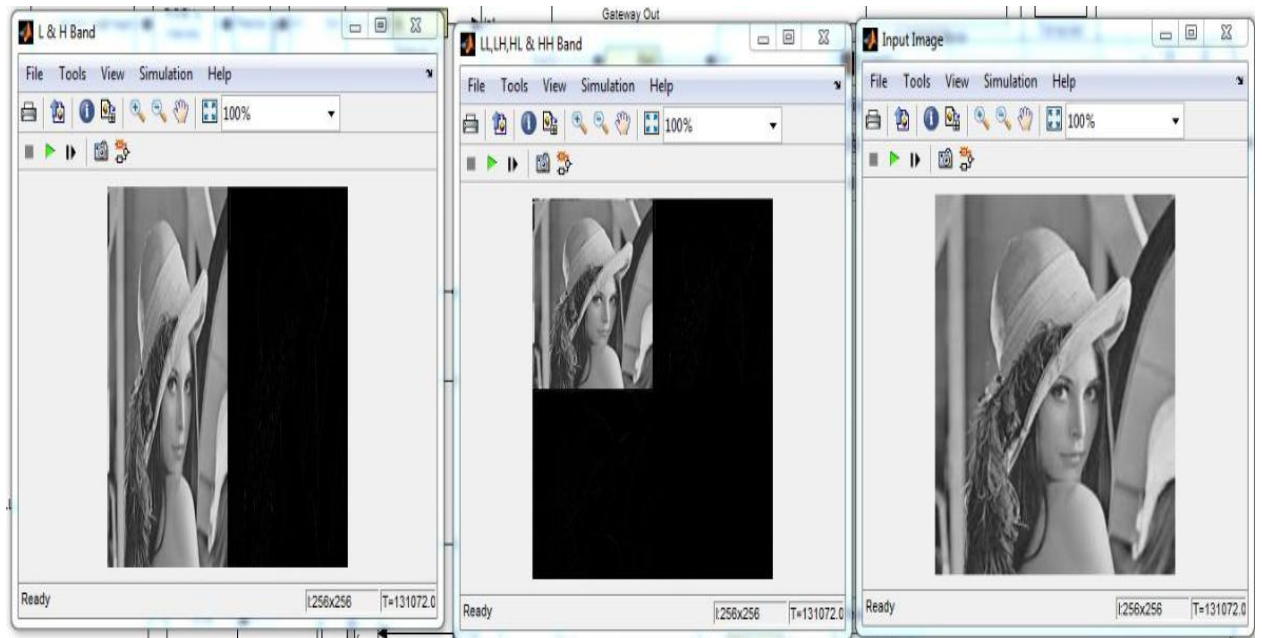

Figure 5: Hardware output (Image) from 2D-DWT

\section{Conclusion}

The architecture is implemented on Atlys FPGA using System-Generator tool. The output image of the architecture shows that the architecture is efficient to produce the result with accuracy.

\section{References}

[1] Tinku Acharya and Chaitali Chakrabarti, "A Survey on Lifting-Based Discrete Wavelet Transform Architectures", International Journal of VLSI Signal Processing, Springer, Vol. 42, pp. 321-339, 2006.

[2] Kishore Andra, Chaitali Chakrabarti and Tinku Acharya, "A VLSI Architecture for Lifting-Based Forward and Inverse Wavelet Transform”, IEEE Transaction on Signal Processing, Vol. 50, No. 4, pp. 966-977, April 2002.

[3] H. Liao, M. K. Mandal and B. F. Cockburn, "Efficient Architectures for 1-D and 2-D Lifting-Based Wavelet Transforms", IEEE Transaction on Signal Processing, Vol. 52, No. 5, pp. 1315-1326, May 2004.

[4] S. Barua, J. E. Carletta, K. A. Kotteri and A. E. Bell, "An Efficient Architecture for Lifting-Based Two-Dimensional Discrete Wavelet Transform", Integration VLSI Journal, Vol. 38, No. 3, pp. 341-352, 2005

[5] Maria E. Angelopoulou, Peter Y.K. Cheung, Konstantions and Yiannis Andreopouos, "Implementation and Comparison of the 5/3 Lifting 2D Discrete Wavelet Transform Computation Schedules on FPGAs", International Journal of Signal Processing Systems, Springer, Vol.51, pp. 3-21, 2008. 
[6] Xuguang Lan, Nanning Zheng and Yuehu Liu, "Low-Power and High-Speed VLSI Architecture for Lifting-Based Forward and Inverse Wavelet Transform”, IEEE Transactions on Consumer Electronics, Vol. 51, No. 2, pp. 607-613, May 2005.

[7] Yeong-Kang Lai, Lien-Fei Chen and Yui-Chih Shih, "A High-Performance and Memory-Efficient VLSI Architecture with Parallel Scanning Method for 2-D Lifting-Based Discrete Wavelet Transform”, IEEE Transactions on Consumer Electronics, Vol. 55, No. 2, pp. 400-407, May 2009.

[8] P. Wu and L. Chen, "An Efficient Architecture for Two-Dimensional Discrete Wavelet Transform”, IEEE Transaction on Circuits System and Video Technology, Vol. 11, No. 4, pp. 536-545, April 2001.

[9] C. Chakrabarti, M. Vishwanath, and R. M. Owens, "Architectures for wavelet transforms: A survey," Journal of VLSI Signal Processing, vol. 14, no. 2, 1996, pp. 171-192.

[10] K. Masselos, Y. Andreopoulos, and T. Stouraitis, "Performance comparison of two-dimensional discrete wavelet transform computation schedules on a VLIW digital signal processor," in IEE Proceedings Vision, Image \& Signal Processing, vol. 153, no. 2, 2006, pp. 173-180.

[11] M. Angelopoulou, K. Masselos, P. Cheung, and Y. Andreopoulos, "A Comparison of 2-D Discrete Wavelet Transform Computation Schedules on FPGAs," in IEEE International Conference on Field Programmable Technology, 2006, pp. 181-188.

[12] N. D. Zervas, G. P. Anagnostopoulos, V. Spiliotopoulos, Y. Andreopoulos, and C. E. Goutis, "Evaluation of design alternatives for the 2-D-discrete wavelet transform," IEEE Transactions on Circuits and Systems for Video Technology, vol. 11, no. 12, 2001, pp. $1246-1262$.

[13] Shilpa and Meghana Kulkarni, "An Efficient VLSI Implementation of CDF 5/3 Architecture on FPGA for Image Processing Application", IJSTE, Vol. 2, Issue 12, pp. 329-332, 2016. Steven T. Karris, "Introduction to Simulink with Engineering Applications", Orchad Publications, 2006. 